\title{
Contact Angles of Sessile Droplets Deposited on Rough and Flat Surfaces in the Presence of External Fields
}

\author{
E. Bormashenko ${ }^{1 *}$ \\ ${ }^{1}$ Ariel University Center of Samaria, Applied Physics Department, Department of Chemistry \\ and Biotechnology Engineering, POB 3, Ariel, 40700, Israel
}

\begin{abstract}
The paper proposes a general framework allowing the analysis of wetting problems in the situation when interfacial tensions depend on external fields. An equation predicting apparent contact angles of sessile droplets deposited on rough surfaces in the presence of external fields is derived. The problem of wetting is discussed in the framework of the variational approach. Derivation of a general equation generalizing the Cassie and Wenzel approaches is presented. The effects related to the line tension which are important for nano-structured surfaces are considered.
\end{abstract}

Keywords and phrases: electrowetting, dependence of interfacial tensions on external fields Cassie and Wenzel models, line tension, excess free energy variation, transversality conditions

Mathematics Subject Classification: $76 \mathrm{~T} 10$

The phenomena of electro- magneto- and optowetting have attracted the attention of researchers recently. These effects have been applied for various microfluidics applications including smart actuation of droplets and micro-droplets [1-8]. A smart actuation of droplets deposited on nano-structured surfaces is of particular interest. Krupenkin et al demonstrated the dynamic electrical control of the wetting behavior of liquids on nanostructured surfaces, which spans the entire possible range from superhydrophobic behavior to nearly complete wetting [4]. Electro-, magneto- and optowetting techniques exploit the dependence of interfacial tensions on external fields [7]. A general approach to the calculation of contact angles of droplets deposited on flat surfaces in the presence of external fields is given in Ref. [9]. Below, a general theoretical framework is presented, which allows calculation of apparent contact angles of droplets deposited on a rough surface and exposed to an external field. The Cassie and Wenzel models [10-13] are believed to give the theoretical background of wetting of rough surfaces. The Wenzel model implies the complete wetting of a rough surface, i.e. liquid penetrates into grooves constituting the surface; whereas the Cassie wetting describes the situation where a droplet partially wets the rough surface and sits on air pockets [10-13]. The Wenzel and Cassie models helped to explain a considerable diversity of wetting phenomena occurring on rough surfaces. However, these models have been criticized recently and gener-

*Corresponding author. E-mail: edward@ariel.ac.il

(C) EDP Sciences, 2012 
alized over the past decade [14-18]. Below, a generalization of the Cassie and Wenzel models is presented for the situation when the interfacial tension depends on an applied external field.

\section{Results and discussion}

We discuss an axially symmetric droplet of the radius a (see Figure 1) containing liquid, deposited on a solid rough surface and surrounded by a gaseous phase. The system is exposed to an external field (it may be an electric or a magnetic field, without loss of generality of our treatment). It is supposed that the most general regime of wetting takes place, i.e. the so-called mixed wetting occurs. This means that the droplet partially wets the solid surface and is partially supported by air pockets, as shown in Figure 1. It is assumed that the effects related to line tension are not negligible [19]. The apparent contact angle of the droplet will be governed by the topography of the substrate, the triad of surface tensions $\gamma, \gamma_{S L}, \gamma_{S A}$, which are the interfacial tensions at the liquid/gaseous phase, solid/liquid and solid/air interfaces, respectively, and the line tension $\Gamma[10]$ (which is assumed to be field-independent). All the interfacial tensions are assumed to be field-dependent for the sake of generality. In the majority of experimental situations $\gamma_{S L}$ is field-dependent. This is typical for electro-wetting [8-10]; however, the cases where $\gamma$ and $\gamma_{S A}$ were influenced by an external field have also been reported $[7,20]$. For the present axially symmetric case, it is anticipated that the interaction of the droplet with the field is described by the linear density $U(h, x)$ of the additional energy with the dimension of $(\mathrm{J} / \mathrm{m})$

$$
U(h(x), x)=\int_{0}^{h(x)} 2 \pi x w(x, y) d y
$$

where $w(x, y)$ is the volume energy density of the droplet bulk in the external field, and $h(x)$ is the local height of the liquid surface above the substrate (see Figure 1). The functions $w(x, y)$ and $U(h(x), x)$ are determined by the external field and are supposed to be known. I suggest also that the interfacial tensions could be expressed as explicit functions of the coordinates: $\gamma=\gamma(x, y), \gamma_{S L}=\gamma_{S L}(x, y), \gamma_{S A}=\gamma_{S A}(x, y)$. The types of these functions are governed by the spatial distribution of the external field and are also supposed to be known. The free energy $G$ of the axially symmetric droplet exposed to the axially symmetric field is given by:

$$
\begin{gathered}
G=\int_{0}^{a}\left(\gamma(x, h) 2 \pi x \sqrt{1+\left(\frac{d h}{d x}\right)^{2}}+\right. \\
\left.2 \pi x\left(\gamma_{S L}(x, 0)-\gamma_{S A}(x, 0)\right) r \Phi_{S}+2 \pi x \gamma(x, 0)\left(1-\Phi_{S}\right)+2 \pi \Gamma+2 \pi x \Gamma \xi+U(h, x)\right) d x,
\end{gathered}
$$

where $\Phi_{S}$ is the fraction of the solid surface that is wetted by the liquid, $r$ is the roughness ratio of the wetted area, and $\xi$ is the perimeter of the triple line per unit area of the substrate under the droplet (with the dimension of $m^{-1}$, see Ref. [19]). It is suggested that the radius of the droplet $a$ is much larger than the characteristic scale of the substrate roughness [15]. In Eq. (1)

$$
\int_{0}^{a}\left(\gamma(x, h) 2 \pi x \sqrt{1+\left(\frac{d h}{d x}\right)^{2}}\right) d x
$$

presents the energy of the liquid/air cap interface,

$$
\int_{0}^{a}\left(2 \pi x\left(\gamma_{S L}(x, 0)-\gamma_{S A}(x, 0)\right) r \Phi_{S}\right) d x
$$

is the energy of the solid wetted with liquid, $\int_{0}^{a}\left(2 \pi x \gamma(x, 0)\left(1-\Phi_{S}\right)\right) d x$ is the energy of the liquid/air interface over air pockets, $\int_{0}^{a}(2 \pi \Gamma) d x$ is the energy of the external triple line surrounding the droplet, $\int_{0}^{a}(2 \pi x \Gamma \xi) d x$ is the energy of internal triple lines (see Figure 1, depicting a droplet deposited on a 
substrate built of conical posts; the "external" and "internal" triple lines are shown). The condition of the constant volume $\mathrm{V}$ of the droplet also has to be taken into account in solving a variational problem:

$$
V=\int_{0}^{a}(2 \pi x h(x) d x)=\text { const. }
$$

Equations (1) and (2) reduce the problem to a minimization of the functional:

$$
\begin{gathered}
G=\int_{0}^{a}\left(\tilde{G}\left(h, h^{\prime}, x\right) d x\right), \\
\tilde{G}=2 \pi \gamma(x, h) x \sqrt{1+h^{\prime 2}}+2 \pi x \Pi(x)+2 \pi \lambda x h+U(h, x)+2 \pi \Gamma,
\end{gathered}
$$

where $\lambda$ is the Lagrange multiplier to be deduced from Eq. (2), and $\Pi(x)=\left(\gamma_{S L}(x, 0)-\gamma_{S A}(x, 0)\right) r \Phi_{S}+$ $\gamma(x, 0)\left(1-\Phi_{S}\right)+\Gamma \xi$. The constant term $2 \pi \Gamma$ in Eq. (4) can be omitted when the functional $G$ is minimized; however, it turns out to be important for the analysis of boundary conditions. It has to be emphasized that I solve the variational problem with free endpoints. Thus, the conditions of transversality have to be considered [21]. It has been already demonstrated that the use of the transversality conditions for the analysis of wetting problems is extremely effective [22-27]. In our case, the transversality condition is given by Eq. (5):

$$
\left(\tilde{G}-h^{\prime} \tilde{G}_{h^{\prime}}^{\prime}\right)_{x=a}=0 .
$$

Substitution of Exp. (4) into Eq. (5), simple transformations akin to those carried out in Refs. [24-27], and considering $h(a)=0 ; U(h=0)=0$, yield:

$$
\left(\frac{\gamma(a, 0) a}{\sqrt{1+h^{\prime 2}}}\right)_{x=a}=(-a \Pi(x)-\Gamma)_{x=a} .
$$

Taking into account $h^{\prime}(x=a)=-\tan \theta^{*}$, where $\theta^{*}$ is the apparent contact angle, immediately gives rise to:

$$
\cos \theta^{*}=-\frac{\Pi_{x=a}}{\gamma(a, 0)}-\frac{\Gamma}{\gamma(a, 0) a} .
$$

Substitution of $\Pi_{x=a}=\left(\gamma_{S L}(a, 0)-\gamma_{S A}(a, 0)\right) r \Phi_{S}+\gamma\left(1-\Phi_{S}\right)+\Gamma \xi$ into Eq. (7) yields:

$$
\cos \theta^{*}=\frac{\gamma_{S A}(a, 0)-\gamma_{S L}(a, 0)}{\gamma(a, 0)} r \Phi_{S}+\Phi_{S}-1-\frac{\Gamma}{\gamma(a, 0)}\left(\xi+\frac{1}{a}\right) .
$$

It can be recognized that only the values of interfacial tensions at the endpoints (in other words at the triple line) govern the contact angle. It is also noteworthy that the apparent contact angle $\theta^{*}$ does not depend explicitly on the linear density of the external field $U(h, x)$; however, the external field dictates the types of $\gamma=\gamma(x, 0), \gamma_{S L}=\gamma_{S L}(x, 0)$, and $\gamma_{S A}=\gamma_{S A}(x, 0)$ [24-27]. Now let us discuss special cases of wetting. When the substrate is flat $\left(r=1, \Phi_{S}=1\right)$, and the effects related to line tension are negligible (the droplet is sufficiently large; the effects of line tension become considerable for micro-metrically scaled drops or nano- and micro-structured surfaces [10, 19, 28]), we obtain:

$$
\cos \theta^{*}=\frac{\gamma_{S A}(a, 0)-\gamma_{S L}(a, 0)}{\gamma(a, 0)}
$$

When $\gamma, \gamma_{S A}=$ const, $\gamma_{S L}=\gamma_{S L}^{0}-\frac{C V^{2}}{2}(C$ - the capacitance, $V$ - voltage $)$ we obtain the well-known equation of electrowetting $[8,9]$ :

$$
\cos \theta^{*}=\frac{\gamma_{S A}-\gamma_{S L}^{0}+\frac{C V^{2}}{2}}{\gamma}=\cos \theta_{Y}+\frac{C V^{2}}{2 \gamma},
$$


where $\theta_{Y}$ is the Young angle characterizing a solid/liquid pair. When $r=1$ and the effects related to the line tension are negligible, we obtain the modified Cassie-Baxter equation considering the field dependencies of the interfacial tensions:

$$
\cos \theta^{*}=\frac{\gamma_{S A}(a, 0)-\gamma_{S L}(a, 0)}{\gamma(a, 0)} \Phi_{S}+\Phi_{S}-1
$$

When $\Phi_{S}=1$, and the effects related to the line tension are negligible, we obtain the modified Wenzel equation:

$$
\cos \theta^{*}=\frac{\gamma_{S A}(a, 0)-\gamma_{S L}(a, 0)}{\gamma(a, 0)} r .
$$

When the effects related to the line tension are negligible, and so-called mixed wetting occurs, $r \neq 1$, and we obtain the modified Miwa-Marmur equation $[14,16]$ :

$$
\cos \theta^{*}=\frac{\gamma_{S A}(a, 0)-\gamma_{S L}(a, 0)}{\gamma(a, 0)} r \Phi_{S}+\Phi_{S}-1
$$

It also should be mentioned that, as is seen from the above equations, only the area adjacent to a triple line exerts an influence on the apparent contact angle. Apparent contact angles are governed by interfacial tensions at the triple line and the geometrical parameters of defects $\Phi_{S}, \xi, r$ located in the vicinity of the three-phase (triple) line [26].

\section{Conclusions}

A general approach allowing the prediction of the apparent contact angle of a droplet deposited on a rough surface and exposed to an external field is proposed. The approach is based on the use of transversality conditions of an appropriate variational problem of wetting. The values of interfacial tensions at the triple line and the topography of the surface in the vicinity of the triple line govern the apparent contact angle. The equation describing Cassie, Wenzel and Miwa-Marmur wetting of sessile droplets exposed to external fields is reported.

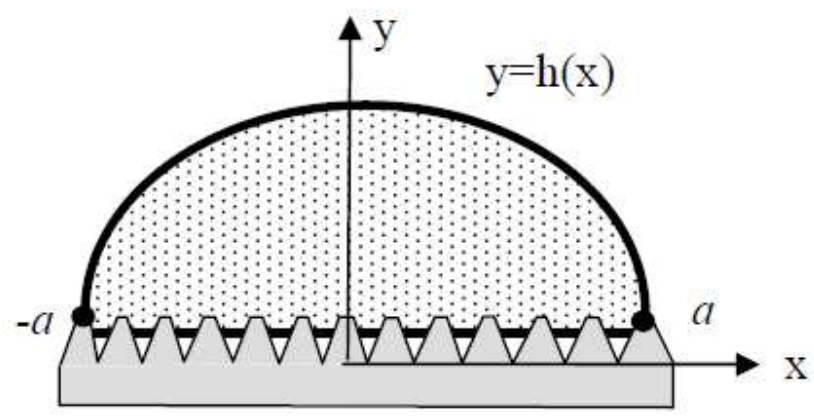

FiguRE 1. Mixed wetting of a rough surface. Interfacial tensions are spatially dependent: $\gamma=\gamma(x, y), \gamma_{S L}=\gamma_{S L}(x, y), \gamma_{S A}=\gamma_{S A}(x, y)$. 
Acknowledgements. The author is grateful to Dr. Gene Whyman for fruitful discussions. The author is thankful to Mrs. Ye. Bormashenko and Mrs. Al. Musin for their kind help in preparing this manuscript.

\section{References}

[1] M. Pollack, R. Fair, A. Shenderov. Electrowetting-based actuation of liquid droplets for microfluidic applications. Appl. Phys. Lett., 77 (2000), 1725-1726.

[2] R. Hayes, B. Feenstra. Video-speed electronic paper based on electrowetting. Nature, 425 (2003), 383-385.

[3] Fr. Mugele, J.-Ch. Baret. Electrowetting: from basics to applications. J. Phys.: Condens. Matter, 17 (2005), R705-R774.

[4] T. Krupenkin, J. Taylor, T. Schneider, S. Yang.From Rolling Ball to Complete Wetting: The Dynamic Tuning of Liquids on Nanostructured Surfaces. Langmuir, 20 (2004), 3824-3827.

[5] N.-Tr. Nguyen, G. Zhu, Y.-Ch. Chua, V.-Ng. Phan, S.-H. Tan. Magnetowetting and Sliding Motion of a Sessile Ferrofluid Droplet in the Presence of a Permanent Magnet. Langmuir, 26 (2010), 12553-12559.

[6] Q. Zhou, W. Ristenpart, P. Stroeve. Magnetically Induced Decrease in Droplet Contact Angle on Nanostructured Surfaces. Langmuir 27, (2011), 11747-11751.

[7] L. Liggieri, A. Sanfeld, A. Steinchenbad. Effects of magnetic and electric fields on surface tension of liquids. Physica A, 206 (1994), 299-331.

[8] A. Banpurkar, K. Nichols, Fr. Mugele. Electrowetting-Based Microdrop Tensiometer. Langmuir, 24 (2008), 1054910551.

[9] B. Shapiro, H. Moon, R. Garrell, CJ. Kim. Equilibrium behavior of sessile drops under surface tension, applied external fields, and material variations. J. Applied Physics, 93 (2003), 5794-5811

[10] P. de Gennes, F. Brochard-Wyart, D. Quere. Capillarity and Wetting Phenomena. Springer, Berlin, 2003.

[11] A. Cassie, S. Baxter. Wettablity of porous surfaces. Trans. Faraday Soc., 40 (1944), 546-551.

[12] A. Cassie. Contact angles. Discuss. Faraday Soc., 3 (1948), 11-16.

[13] R. Wenzel. Resistance of solid surfaces to wetting by water. Ind. Eng. Chem., 28 (1936), 988-994.

[14] A. Marmur. Wetting on hydrophobic rough surfaces: to be heterogeneous or not to be? Langmuir, 19 (2003), 8343-8348.

[15] M. Nosonovsky. On the Range of Applicability of the Wenzel and Cassie Equations. Langmuir, 23 (2007), 9919-9920.

[16] M. Miwa, A. Nakajima, A. Fujishima, K. Hashimoto, T. Watanabe. Effects of the Surface Roughness on Sliding Angles of Water Droplets on Superhydrophobic Surfaces. Langmuir, 16 (2000), 5754.

[17] S. Larsen, R. Taboryski. A Cassie-like law using triple phase boundary line fractions for faceted droplets on chemically heterogeneous surfaces. Langmuir, 25 (2009), 1282-1284.

[18] B. Bhushan; M. Nosonovsky. The rose petal effect and the modes of superhydrophobicity. Philosophical Trans. Royal Soc. A, 368 (2010), 4713-4728.

[19] T.-S. Wong, Ch.-M. Ho. Dependence of Macroscopic Wetting on Nanoscopic Surface Textures. Langmuir, 25 (2009), 12851-12854.

[20] D. Aronov, M. Molotskii, G. Rosenman. Electron-induced wettability modification. Physical Review B 76 (2007), 035437.

[21] I. Gelfand, S. Fomin. Calculus of Variations. Dover, New York, 2000.

[22] A. Marmur. Line tension effect on contact angles: Axisymmetric and cylindrical systems with rough or heterogeneous solid surfaces. Colloids Surf. A, 136 (1998), 81-88.

[23] V. Starov, M. Velarde. Surface forces and wetting phenomena. J. Phys. Condens. Matter., 21 (2009), 464121.

[24] E. Bormashenko. Young, Boruvka-Neumann, Wenzel and Cassie-Baxter equations as the transversality conditions for the variational problem of wetting. Colloids and Surfaces A, 345 (2009), 163-165.

[25] E. Bormashenko. Wetting of Flat and Rough Curved Surfaces. J. Phys. Chem. C, 113 (2009), 17275-17277.

[26] E. Bormashenko. A Variational Approach to Wetting of Composite Surfaces: Is Wetting of Composite Surfaces a One-Dimensional or Two-Dimensional Phenomenon? Langmuir, 25 (2009), 10451-10454.

[27] E. Bormashenko. General equation describing wetting of rough surfaces. Journal of Colloid and Interface Science, 360 (2011), 317-319.

[28] A. Amirfazli, A. Neumann. Status of three-phase line tension. Advances in Colloid and Interface Science, 110 (2004), $121-141$. 